\title{
The Cosmic Lens All-Sky Survey - I. Source selection and observations
}

\author{
S. T. Myers, ${ }^{1,2 \star}$ N. J. Jackson, ${ }^{3}$ I. W. A. Browne, ${ }^{3}$ A. G. de Bruyn, ${ }^{4,5}$ T. J. Pearson, ${ }^{6}$ \\ A. C. S. Readhead,${ }^{6}$ P. N. Wilkinson, ${ }^{3}$ A. D. Biggs, ${ }^{3}$ R. D. Blandford, ${ }^{6}$ \\ C. D. Fassnacht, ${ }^{1,6,7}$ L. V. E. Koopmans, ${ }^{3,4,6}$ D. R. Marlow, ${ }^{2,3}$ J. P. McKean, ${ }^{3}$ \\ M. A. Norbury, ${ }^{3}$ P. M. Phillips, ${ }^{3}$ D. Rusin, ${ }^{2,8}$ M. C. Shepherd ${ }^{6}$ and C. M. Sykes ${ }^{3}$ \\ ${ }^{1}$ National Radio Astronomy Observatory, PO Box O, Socorro, NM 87801, USA \\ ${ }^{2}$ Department of Physics and Astronomy, University of Pennsylvania, 209 S. 33rd Street, Philadelphia, PA 19104-6396, USA \\ ${ }^{3}$ University of Manchester, Jodrell Bank Observatory, Macclesfield, Cheshire SK11 9DL \\ ${ }^{4}$ Kapteyn Astronomical Institute, Postbus 800, 9700 AA Groningen, the Netherlands \\ ${ }^{5}$ NFRA, Postbus 2, 7990 AA Dwingeloo, the Netherlands \\ ${ }^{6}$ California Institute of Technology, 105-24, Pasadena, CA 91125, USA \\ ${ }^{7}$ Space Telescope Science Institute, Baltimore, MD 21218, USA \\ ${ }^{8}$ Harvard-Smithsonian Center for Astrophysics, 60 Garden Street, MS-51, Cambridge, MA 02138, USA
}

Accepted 2002 November 1. Received 2002 October 23; in original form 2002 July 10

\begin{abstract}
The Cosmic Lens All-Sky Survey (CLASS) is an international collaborative programme which has obtained high-resolution radio images of over 10000 flat-spectrum radio sources in order to create the largest and best-studied statistical sample of radio-loud gravitationally lensed systems. With this survey, combined with detailed studies of the lenses found therein, constraints can be placed on the expansion rate, matter density and dark energy (e.g. cosmological constant, quintessence) content of the Universe that are complementary to and independent of those obtained through other methods. CLASS is aimed at identifying lenses where multiple images are formed from compact flat-spectrum radio sources, which should be easily identifiable in the radio maps. Because CLASS is radio-based, dust obscuration in lensing galaxies is not a factor, and the relative insensitivity of the instrument to environmental conditions (e.g. weather, 'seeing') leads to nearly uniform sensitivity and resolution over the entire survey. In four observing 'seasons' from 1994 to 1999, CLASS has observed 13783 radio sources with the Very Large Array (VLA) at $8.4 \mathrm{GHz}$ in its largest 'A'-configuration (0.2-arcsec resolution). When combined with the Jodrell Bank VLA Astrometric Survey (JVAS), the CLASS sample contains over 16000 images. A complete sample of 11685 sources was observed, selected to have a flux density of at least $30 \mathrm{mJy}$ in the Green Bank Survey (GB6) catalogue at $4.85 \mathrm{GHz}$ (spanning the declination range $0^{\circ} \leqslant \delta \leqslant 75^{\circ}$ and $|b| \geqslant 10^{\circ}$, excluding the Galactic plane) and a spectral index $\alpha \geqslant-0.5$ between the NRAO-VLA Sky Survey (NVSS) at $1.4 \mathrm{GHz}$ and the GB6. A typical 30-s CLASS snapshot reached an rms noise level of $0.4 \mathrm{mJy}$. So far, CLASS has found 16 new gravitational lens systems, and the JVAS/CLASS survey contains a total of 22 lenses. The follow-up of a small number of candidates using the VLA, MERLIN, the Very Long Baseline Array (VLBA) and optical telescopes is still underway. In this paper, we present a summary of the CLASS observations, the JVAS/CLASS sample, and statistics on sub-samples of the survey. An accompanying paper presents the lens candidate selection, and in a third paper the implications for cosmology are discussed.
\end{abstract}

Key words: gravitational lensing - cosmology: observations.

\section{INTRODUCTION}

The Cosmic Lens All-Sky Survey (CLASS) is an international programme to map more than 10000 radio sources in order to create

^E-mail: smyers@nrao.edu the largest and best-studied statistical sample of radio-loud gravitationally lensed systems. CLASS is aimed at identifying lenses where multiple images are formed from compact flat-spectrum radio sources. The resulting lens configurations should be easily identifiable in the radio maps. CLASS is most efficient at finding galaxy-mass lenses with arcsecond image separations, as these mass 
distributions dominate the lensing optical depth. In principle, CLASS can detect more extreme lensed systems with larger separations arising from clusters of galaxies (Phillips et al. 2001), as well as smaller-separation (160-300 mas) lenses (Augusto, Wilkinson \& Browne 1998; Augusto et al. 2001). Setting limits on gravitational lensing on these smaller and larger angular scales is a secondary goal of the survey.

The Very Large Array (VLA) was used as the primary instrument for the CLASS survey. In its largest 'A'-configuration, the VLA provides high-quality snapshot images with angular resolution of $0.2-0.3$ arcsec at an average observing frequency of $8.46 \mathrm{GHz}$. In the four primary phases of CLASS carried out from 1994 to 1999 , a total of 13783 target radio sources were mapped. From among these, hundreds of multiple-component sources were identified and followed up with higher resolution using the Multi-Element RadioLinked Interferometer Network (MERLIN) (50-mas resolution) and the Very Long Baseline Array (VLBA) (2-mas resolution), and with multiwavelength observations using a number of instruments. So far, CLASS has discovered 16 new radio-loud gravitational lens systems, which when added to the six lenses from the Jodrell Bank VLA Astrometric Survey (JVAS) makes a total of 22 confirmed lens systems in JVAS/CLASS (Table 1). Some follow-up observations are still underway but it is doubtful that more multiple-image systems will be found in the sample. For example, we have yet to identify conclusively the CLASS-2 source CLASS B0827+525, a 2.82-arcsec double, as either a 'dark lens' or a binary radio-loud quasar (Koopmans et al. 2000b). The details of this follow-up programme and the verified lenses are given in Paper II (Browne et al. 2003).

There have been previous VLA-based lens surveys: the MITGreen Bank (MG) survey (Burke 1989), and the JVAS survey (Patnaik et al. 1992a; Browne et al. 1998; Wilkinson et al. 1998).
JVAS sources, like CLASS sources, were required to have flat radio spectra, but the JVAS survey was restricted to 5-GHz radio flux densities $\geqslant 200 \mathrm{mJy}$, compared with the 25-30 mJy limit of CLASS. JVAS is thus effectively a bright sub-sample of CLASS. The main difference between JVAS/CLASS and the MG survey is that the latter has no spectral index selection, and so also picks up sources dominated by steep-spectrum lobe emission. Of the $\sim 5000$ target sources observed in the two previous surveys, five multiply imaged lens systems were found in the MG survey and six in JVAS, of which one was a rediscovery of one of the MG lenses. CLASS has more than doubled the number of radio-discovered lens systems known, and JVAS/CLASS as a whole constitutes the most comprehensive single sample of lenses for use in astrophysical applications. In addition to these northern celestial hemisphere surveys, the VLA is also being used to carry out a gravitational lens survey in the southern sky (Winn et al. 2000).

One of the most important uses of galaxy-mass lenses is in the determination of cosmological parameters. In particular, time delays measured between the components in multiple-image systems with reliable mass models can directly determine the angular diameter distances to the lens and lensed objects, and therefore the Hubble constant $H_{0}$. Because the lensing equations depend on a ratio of distances, the delays are not as sensitive to the matter density $\Omega_{\mathrm{m}}$ and cosmological constant $\Lambda_{0}$. Of course, the success of the method depends upon the presence of measurable variability in the lensed source, and the ability to construct a well-constrained mass model for the lens, which in turn should ideally consist of a single deflector well fitted by a spherical or ellipsoidal potential. Compact flat-spectrum lensed radio sources are more likely to be variable and are easily recognized as multiply imaged. Steepspectrum, extended lensed sources can provide more constraints on the lensing potential, but are unlikely to be variable and are hard

Table 1. JVAS/CLASS lenses. The separation for lenses with more than two components is the maximum separation. The number of images listed is the number of radio components believed to be lensed images, not counting sub-components seen on VLBI scales. The principal references are given for each lens. The 13 lenses from the CLASS complete sample (Section 2.2) are shown in boldface.

\begin{tabular}{|c|c|c|c|c|}
\hline Lens & Session & $\begin{array}{c}\text { Max. Sep. } \\
(\operatorname{arcsec})\end{array}$ & No. of images & References \\
\hline B0128+437 & CLASS-1 & 0.54 & 4 & Phillips et al.(2000) \\
\hline B0218+357 & JVAS & 0.33 & $2+\operatorname{ring}$ & Patnaik, Porcas \& Browne (1995); Biggs et al. (1999) \\
\hline MG0414+054 & MG, JVAS & 2.09 & 4 & Hewitt et al. (1992) \\
\hline B0445+123 & CLASS-2 & 1.33 & 2 & Argo et al. (2003) \\
\hline B0631+519 & CLASS-1 & 1.16 & 2 & York et al. (in preparation) \\
\hline B0712+472 & CLASS-1 & 1.27 & 4 & Jackson et al. (1998) \\
\hline B0739+366 & CLASS-2 & 0.54 & 2 & Marlow et al. (2001) \\
\hline B0850+054 & CLASS-3 & 0.68 & 2 & Biggs et al. (2003) \\
\hline B1030+074 & JVAS & 1.56 & 2 & Xanthopoulos et al. (1998) \\
\hline B1127+385 & CLASS-2 & 0.70 & 2 & Koopmans et al. (1998) \\
\hline B1152+199 & CLASS-3 & 1.56 & 2 & Myers et al. (1999); Rusin et al. (2002) \\
\hline B1359+154 & CLASS-3 & 1.65 & 6 & Myers et al. (1999); Rusin et al. (2001b) \\
\hline $\mathrm{B} 1422+231$ & JVAS & 1.28 & 4 & Patnaik et al. (1992b) \\
\hline B1555+375 & CLASS-2 & 0.43 & 4 & Marlow et al. (1999) \\
\hline B1600+434 & CLASS-1 & 1.39 & 2 & Jackson et al. (1995) \\
\hline $\mathrm{B} 1608+656$ & CLASS-1 & 2.08 & 4 & Myers et al. (1995); Snellen et al. (1995) \\
\hline $\mathrm{B} 1933+503$ & CLASS-1 & 1.17 & $4+4+2$ & Sykes et al. (1998) \\
\hline B1938+666 & JVAS & 0.93 & $4+$ ring & King et al. (1997) \\
\hline B2045+265 & CLASS-2 & 1.86 & 4 & Fassnacht et al. (1999a) \\
\hline B2108+213 & CLASS-4 & 4.60 & 2 & McKean et al. (in preparation) \\
\hline B2114+022 & JVAS & 2.57 & 4 & Augusto et al. (2001); Chae, Mao \& Augusto (2001) \\
\hline B2319+051 & CLASS-2 & 1.36 & 2 & Rusin et al. (2001a) \\
\hline
\end{tabular}


to distinguish from complex unlensed radio lobes. Thus CLASS is aimed at filtering out the latter in preference for the former. Unfortunately, only a few lenses found in CLASS will satisfy these criteria and be suitable as cosmological standards. The CLASS group has successfully monitored the quadruple lens CLASS B1608+656 (Fassnacht et al. 1999b; Koopmans \& Fassnacht 1999; Fassnacht et al. 2002), and all three independent time delays between the four components have been measured. For the current standard cosmology $\left(\Omega_{\mathrm{m}}=0.3, \Lambda_{0}=0.7\right)$, a Hubble constant $H_{0}=61-65$ (galaxy centroid positions) \pm 2 ( $2 \sigma$ statistical) \pm 15 (systematic) $\mathrm{km}$ $\mathrm{s}^{-1} \mathrm{Mpc}^{-1}$ has been derived (Fassnacht et al. 2002). In addition, the double-image systems CLASS B1600+434 (Koopmans et al. 2000a; Koopmans \& de Bruyn 2000) and JVAS B0218+357 (Biggs et al. 1999) were monitored and time delays measured, giving Hubble constant values of $57_{-11}^{+14}$ and $69_{-11}^{+13} \mathrm{~km} \mathrm{~s}^{-1} \mathrm{Mpc}^{-1}$ respectively (errors are $2 \sigma$ statistical errors assuming singular isothermal ellipsoid mass models). The mass profile modelling for these systems is being further refined, and new results will be presented in upcoming papers.

The observed lensing rate, and to a lesser extent the distribution of image separations and redshifts of the lenses, provides constraints upon the cosmology, particularly the differential volume of the universe versus redshift, which is largely controlled by the matter density $\Omega_{\mathrm{m}}$ and the dark energy density $\Lambda_{0}$ and its equation of state parameter $w$ ranging between $w=-1$ for a cosmological constant and $w=0$ for non-relativistic matter. A well-defined sample selection is necessary to understand the statistics of the lensing, which is in turn necessary to constrain $\Lambda_{0}$ (Turner et al. 1984; Kochanek 1996). For this purpose it is vital that all the lenses in the observed sample must be identified. Because CLASS is radio-based, complications arising from dust obscuration in lensing galaxies do not occur; and because it targets flat-spectrum radio sources with intrinsically simple radio structures, real lenses are easy to identify. Furthermore, the external conditions do not strongly affect the observations and the survey was carried out with nearly uniform sensitivity and resolution. All these factors make it the best sample overall for statistical studies of lenses. However, interpretation of the statistical results from any observational survey in the context of theoretical models requires the control of selection effects in the data sample. In the case of CLASS, this means studying the effects of the lensing on the parent sample of the survey, the resulting image geometries, the ability of the automatic mapping procedure to identify the lensed images, and possible loss of lenses from the survey. In addition, the CLASS sources suffer from the drawbacks generic to radio-selected samples of having poorly constrained redshift distributions and source luminosity functions, which must be dealt with both statistically and through follow-up optical observations.

Data from CLASS also provide important astrophysical constraints upon the mass distribution within the lensing galaxies themselves (e.g. Kochanek 1995). Koopmans \& de Bruyn (2000) observed the signatures of microlensing in the radio light curves of the two images in the system CLASS B1600+434, and deduced that a significant fraction of the mass in the inner dark matter halo of the lensing galaxy is in the form of compact objects. Rusin \& Ma (2001) used the absence of faint 'odd' images in deep radio maps of CLASS lenses to place a lower limit on the inner mass profiles of lensing galaxies. Rusin \& Tegmark (2001) considered the frequency of quadruple-image systems in CLASS and attempted to derive a consistent set of astrophysical mechanisms that explain this statistic as well as other observational constraints. Keeton \& Madau (2001) and Phillips et al. (2001) constrained the concen- tration of cluster-mass dark matter haloes based on the paucity of wide-separation lens systems in the CLASS sample. Cohn et al. (2001) used the lensing model for CLASS B1933+503 to constrain density profiles. Rusin et al. (2002) used high-resolution radio and optical observations of CLASS B1152+199 to model the galaxy mass profile. Finally, observations of the six-image system CLASS B1359+154 were used to place constraints on the mass distribution within the small group of galaxies responsible for the lensing (Rusin et al. 2001b). Astrophysical applications of lensing such as these studies are as important as the cosmological uses outlined above, and may ultimately provide critical clues to the nature and properties of dark matter in galaxies and groups.

The success of the CLASS survey, and the unprecedented observing and analysis speed, was made possible through automation of the observation scheduling and mapping analysis pipeline. Specialized automapping software (see Section 3 ) was developed to carry out these tasks. This has allowed us to observe an average of one target source per minute with the VLA, and then later to map the calibrated data at a similar (now much faster) rate.

In addition to the lens candidates and lensing-related applications, the CLASS survey provides a solid body of radio information for all the objects. Astrometric (35 mas) positions for the sample are determined, which extends the MERLIN/VLBA phase-reference source network in the northern sky to a spacing of one to a few degrees (except in the Galactic plane). CLASS has also proven to be useful in studies of active galactic nuclei. For example, JVAS and CLASS have been used to identify samples of high-redshift quasars (Snellen et al. 2001, 2002). CLASS observations have supplemented lower frequency surveys in identifying young radio sources (Snellen et al. 2000). Marchã et al. (2001) used CLASS to identify a large sample of low-luminosity blazars.

The JVAS/CLASS survey is now complete. This is the first in a series of papers which describe the survey and its results, and covers the initial observations, data reduction and properties of the survey. A second companion paper (Browne et al. 2003, Paper II) describes the selection and follow-up of lens candidates. A third paper (Chae et al. 2002) presents the cosmological results, in particular the derived constraints on the matter density and the dark energy density and its equation of state.

\section{THE SURVEY SAMPLE}

To achieve our goal of obtaining $\sim 20$ new simple lenses suitable for cosmological measurements, we must start with a parent sample of more than 10000 flat-spectrum sources. Moreover, to be easily followed up with higher resolution radio interferometers such as MERLIN, the National Radio Astronomy Observatory (NRAO) VLBA and the European Very Long Baseline Interferometry Network (EVN), the sources should have total compact emission of at least $20 \mathrm{mJy}$. Note that in a lensed system this flux density can be split into four or more images.

To obtain compact multiply imaged components, we target sources with relatively flat radio spectra. We use the convention $S \propto v^{\alpha}$, where $v$ is the frequency of observation. We classify as 'flat-spectrum' those sources with a spectral index of $\alpha \geqslant-0.5$ between $4.85 \mathrm{GHz}$ and a lower frequency. Technically, we start with our 4.85-GHz sample and reject 'steep-spectrum' sources that have $\alpha<-0.5$ versus a lower frequency, as in some stages of the survey targets were kept in the sample when low-frequency measurements were unavailable. 
Table 2. JVAS/CLASS statistics. These numbers reflect pointings that differ by more than 120 arcsec. Duplicate pointings have been excluded. Note that a multiple source will be recorded in cases where bad data or mapping errors have resulted in a spurious secondary. The 'Per cent mult' column refers to the fraction of detected sources with multiple components of any flux density including these spurious multiples - if restricted to $2 \mathrm{mJy}$ or brighter the total is 15.1 per cent of sources detected for all sources and 11.6 per cent in the complete sample.

\begin{tabular}{|c|c|c|c|c|c|c|c|c|}
\hline Session & Dates & $N_{\text {obs }}$ & $N_{\text {det }}$ & Per cent detect & $N_{\text {mult }}$ & Per cent mult & $\mathrm{BW} \mathrm{MHz}$ & Selection \\
\hline JVAS & $1990 \mathrm{Feb}-1992 \mathrm{Dec}$ & 2720 & 2613 & 96.1 & 456 & 17.5 & $25 / 50$ & GB \\
\hline CLASS-1a & 1994 Feb-May & 2550 & 2199 & 86.2 & 644 & 29.3 & 25 & Texas \\
\hline CLASS-1b & $"$ & 670 & 624 & 93.1 & 65 & 10.4 & 50 & WENSS \\
\hline CLASS-2a & 1995 Aug-Sep & 2337 & 2150 & 92.0 & 176 & 8.2 & 50 & WENSS \\
\hline CLASS- $2 b$ & $"$ & 2098 & 1813 & 86.4 & 230 & 12.7 & 25 & Texas/WENSS \\
\hline CLASS-3a & 1998 Feb-May & 2338 & 2043 & 87.4 & 230 & 11.3 & 50 & NVSS \\
\hline CLASS-3b & $"$ & 2058 & 1823 & 88.6 & 249 & 13.7 & 50 & NVSS \\
\hline CLASS-3c & $"$ & 657 & 575 & 87.5 & 125 & 21.7 & 50 & NVSS \\
\hline CLASS-4 & 1999 Aug & 1075 & 753 & 70.0 & 371 & 49.3 & 50 & NVSS \\
\hline Total & & 16503 & 14593 & 88.4 & 2546 & 17.4 & & \\
\hline Complete sample & & 11685 & 10906 & 93.3 & 1558 & 14.3 & & \\
\hline
\end{tabular}

The CLASS observations were taken over 5 years: CLASS-1 in 1994, CLASS-2 in 1995, CLASS-3 in 1998 and CLASS-4 in 1999. Table 2 shows the progression of the survey from JVAS through the end of CLASS. The selection for JVAS is described by Patnaik et al. (1992a). The CLASS survey selection is complicated, and we describe it here in some detail as it affects the use to which the data products may be put. We also define and describe a simpler subset (the CLASS complete sample).

Our starting point for source selection in CLASS-1 and CLASS2 was the $87 \mathrm{~GB}$ version of the Green Bank Survey at $4.85 \mathrm{GHz}$ (Gregory \& Condon 1991). However, the updated survey GB6 (Gregory et al. 1996) became available and was adopted as the parent sample; in this survey the source positions and flux densities are better determined than in $87 \mathrm{~GB}$. Furthermore, in the later stages of the CLASS survey, we adopted the NRAO-VLA Sky Survey (NVSS, Condon et al. 1998) as the low-frequency survey to define the spectral selection. We now describe the observation sessions and the respective selection criteria in detail.

\subsection{CLASS-1 and CLASS-2}

In the first phase of CLASS, observed with the VLA in 1994 February-May, 3220 target fields were mapped. The sources were selected from the 87GB catalogue (Gregory \& Condon 1991) which contains 54579 sources with $4.85-\mathrm{GHz}$ flux densities of $25 \mathrm{mJy}$ or greater in the declination range $0^{\circ} \leqslant \delta \leqslant 75^{\circ}$. We imposed a Galactic latitude limit of $|b| \geqslant 10^{\circ}$. Spectral selection was based on two-point spectral indices versus the 325-MHz Westerbork Northern Sky Survey (WENSS, Rengelink et al. 1997), the 365-MHz Texas Survey (Douglas et al. 1996), and the 1.4-GHz Green Bank Survey (White $\&$ Becker 1992). When available we used the WENSS since the flux densities are reliable and sources have a positional accuracy of $\sim 5$ arcsec. At the time of CLASS-1, only a few 'mini-survey' areas were complete, but by the time of CLASS-2, WENSS was complete above $\delta>30^{\circ}$. In CLASS- 1 and CLASS-2, sources outside the area covered by WENSS at that time were selected using the Texas 365-MHz catalogue.

WENSS has a flux density limit of 15-20 mJy while the 87GB limit is $\sim 25 \mathrm{mJy}$. Target sources were selected with $\alpha \geqslant-0.5$ between $325 \mathrm{MHz}$ and $4.85 \mathrm{GHz}$. There were 670 CLASS-1 sources selected using WENSS (designated as CLASS-1b). The remaining CLASS-1 sources were selected on the basis of flux densities from the Texas $365-\mathrm{MHz}$ catalogue. Because the Texas survey has a flux density limit of $250 \mathrm{mJy}$ at $365 \mathrm{MHz}$, we relaxed the limits to $50 \mathrm{mJy}$ or greater at $4.85 \mathrm{GHz}$ and $\alpha \geqslant-0.6$ between $325 \mathrm{MHz}$ and 4.85 $\mathrm{GHz}$. All sources meeting the criteria were observed for $\delta \geqslant 45^{\circ}$ $20^{\prime}$, and down to $\delta \geqslant 35^{\circ}$ south of the Galactic plane. In all, 2550 sources were observed with this selection (designated CLASS-1a). Note that the Texas Survey only covers the region $\delta<71$.6. North of this declination, there was effectively no spectral selection in CLASS-1a.

In CLASS-2, we were able to select more sources against WENSS as that survey neared completion. In all, 4435 more targets were observed. The CLASS-2a session observed 2337 targets that were nearly all selected using WENSS. The CLASS-2b observations mostly targeted sources below the WENSS southern limit $\left(+30^{\circ}\right)$ and were selected using the Texas Survey and the White \& Becker 1.4-GHz catalogue (which has a flux density limit of $100 \mathrm{mJy}$ at $1.4 \mathrm{GHz}$ ), excluding targets with $\alpha<-0.6$ between $365 \mathrm{MHz}$ or $1.4 \mathrm{GHz}$ and $4.85 \mathrm{GHz}$.

\subsection{CLASS-3, CLASS-4 and the CLASS complete sample}

Our starting point for source selection in CLASS-1 and CLASS2 had been the $87 \mathrm{~GB}$ catalogue. However, the improved Green Bank Survey GB6 became available in 1996. This catalogue contains 75162 sources in the declination range $0^{\circ} \leqslant \delta \leqslant 75^{\circ}$ with a $4.85-\mathrm{GHz}$ flux density of $18 \mathrm{mJy}$ or more and angular diameter 10.5 arcmin or smaller. Since the source positions and flux densities are better determined than in $87 \mathrm{~GB}$, GB6 was adopted as the primary finding list for CLASS-3, with the intention of bringing the entire CLASS survey into line with this new parent catalogue. In addition, the NVSS became available, giving coverage of the entire GB6 region at 1.4 GHz. Use of NVSS created a sample with a uniform rigorous spectral selection over virtually the whole northern sky. This GB6-targeted, NVSS spectrally selected survey will be designated as the 'CLASS-NVSS complete sample' or 'complete sample', and naturally updates the spectral selection used in JVAS as well as early CLASS. We used the NVSS version released as cata$\log 39$.fit for sample selection. This release purported to be complete in the GB6 region (catalog40.fit is now available, filling in holes in other regions of the sky).

The complete sample consists of 11685 targets with a GB6 flux density of $30 \mathrm{mJy}$ or more, an NVSS source within 70 arcsec of the GB6 position, and a spectral index $\alpha \geqslant-0.5$ versus NVSS. The spectral index is defined by adding the flux densities of all NVSS 
sources within 70 arcsec of the GB6 position and ignoring all other NVSS sources. The primary goals of CLASS-3 and CLASS-4 were to complete the sample. A list of 4982 sources for observation in CLASS-3 was drawn up from those sources not within 2 arcmin of a CLASS-1 or CLASS-2 pointing centre or a JVAS target, or identified with a known radio source (e.g. from the VLA calibrator lists).

In addition, the CLASS-3 list was supplemented with a list of GB6 sources (30 mJy or brighter) that have a WENSS source within 70 arcsec of the GB6 position, and spectral index $\alpha \geqslant-0.5$ versus WENSS. This enables the construction of a 'CLASS-WENSS complete sample' and will catch targets that have GHz-peaked spectra where the 1.4-GHz flux density is above the spectral limit for inclusion in the CLASS-NVSS sample.

In total, 5053 new sources were observed in CLASS3 from 1998 February-May. The remaining sources that make up the complete sample were observed in CLASS-4, with a total of 1075 targets observed in 1999 August. Note that most of the CLASS-4 targets were near the lower flux density limit of the sample, entered into the sample on the changeover to the NVSS, or were not detected in NVSS, and thus there is a higher percentage of non-detections and extended or multiple-component sources (see Section 4 and Table 2) in this part of the survey. The final combined CLASS and JVAS archive contains 16503 distinct pointings (more than 120 arcsec apart), of which 11685 form the complete sample. The final complete sample covers an area of $4.96 \mathrm{sr}$.

\section{CLASS VLA OBSERVATIONS AND ANALYSIS}

Two independent intermediate frequencies (IF) bands of width $25 \mathrm{MHz}$ (or $50 \mathrm{MHz}$ with WENSS positions) were centred at 8.415 and $8.465 \mathrm{GHz}$ (average $8.44 \mathrm{GHz}$ ) for CLASS-1 and CLASS-2. In CLASS-3, which was observed in its entirety using NVSS positions and $50-\mathrm{MHz}$ bandwidth, the IF band centres were moved to 8.4351 and $8.4851 \mathrm{GHz}$ (average $8.46 \mathrm{GHz}$ ) in line with NRAO recommendations to avoid radio frequency interference - we will use $8.46 \mathrm{GHz}$ as the fiducial CLASS frequency. For all CLASS observations, an on-source dwell time of $30 \mathrm{~s}$ was used, with 3.3-s integrations. A compact source from the JVAS calibrator list was observed after every $n$th target source for phase calibration, where $n$ was chosen based on the weather conditions prevalent during the session (for CLASS-1: $n=14$; CLASS-2: $n=8$; CLASS-3 $/ 4: n=$ 10-12). The JVAS calibration sources have rms position errors in each coordinate of 12 mas, so the resulting CLASS source positions should have accuracies of about 20 mas (in stable observing conditions) and hence should themselves be useful as potential phase calibration sources. We were able to observe one target source per minute, including the overhead from observing calibration sources and slewing between sources.

The gain calibration using the JVAS astrometric sources was carried out in the Astronomical Image Processing Software (AIPS) package, distributed by NRAO. ${ }^{1}$ The mapping, deconvolution and self-calibration were performed using the DIFMAP package (Shepherd, Pearson \& Taylor 1994; Shepherd 1997). The imaging of the sources was completed in an automated fashion using a DIFMAP script. The average rate for processing with the script was one source every two minutes. This procedure yielded images of good quality for most of the target fields.

\footnotetext{
${ }^{1}$ http://www.cv.nrao.edu/aips
}

The relevant details of the calibration and mapping procedures adopted for CLASS are discussed in the remainder of this section.

\subsection{Calibration and positional accuracy}

Editing and calibration of the data were done using AIPS following the standard procedure. Initial processing was carried out at the time of the observations in order to generate prompt candidate lists for immediate follow-up where possible. After the entire CLASS survey was completed, final consistent editing/calibration was done for the full data set (including JVAS). The data were examined and edited using AIPS TVFLG to remove obviously discrepant telescopes or baselines. The flux density scale was set (AIPS SETJY) using the standard calibrators $3 \mathrm{C} 48$ and 286, for which the flux density scale at $8.41-8.48 \mathrm{GHz}$ in the observations was normalized to the Baars et al. (1977) values. Care was taken to ensure that the flux densities on short baselines of these two sources approximated well to the established values after the final correction was applied; corrections were applied by hand if necessary.

The task AIPS CALIB was used to obtain telescope gain solutions on calibrators, and the resulting solutions were examined. Bad solutions were discarded by hand before interpolation by AIPS CLCAL into a final gain solution. Calibration was applied to the CLASS sources and each CLASS source was split from the file into an output disc UV-FITS file.

15 sources were observed twice during the CLASS-1-3 sessions. This provides a cross-check of the repeatability of our position determinations. These observations were calibrated independently, and did not necessarily use the same phase calibrators. For this set of sources, the rms offset between the positions in the two epochs was found to be 35.2 mas. This is slightly higher than, but consistent with, our expected uncertainties arising from the JVAS position errors.

\subsection{The automapping procedure}

The AUTOMAP algorithm can be outlined as follows. An initial primary cycle is used to find peaks in a large image above some signalto-noise ratio (SNR) cut-off. In a secondary cycle, small images are made around the location of each peak and a CLEAN window is created. In a tertiary cycle, deconvolution and self-calibration are carried out iteratively. The locations of the peaks (and thus the clean boxes) are stored for later use.

A series of parameters define the procedure. The dimensions (cellsize and number of pixels) of the large primary image control the area around the pointing position in which components can be identified. In practice, bandwidth smearing limits our effective field to about 1 arcmin for the 50-MHz observations which form the majority of the sample, and thus a $2048 \times 2048$ pixel search image was constructed with 0.25 -arcsec cell size (giving a $256 \times 256 \operatorname{arcsec}^{2}$ searchable inner quarter) and super-uniform weighting. This field was searched for pixels with values above a given SNR cut-off above which sources are deemed to be reliably identified as real for further processing; SNR $=8$ was adopted for CLASS.

Around each source, a secondary $2048 \times 2048$ image covering $140 \times 140 \operatorname{arcsec}^{2}(0.0684$-arcsec pixels) was then made using uniform weighting, of which the inner quarter $\left(70 \times 70 \operatorname{arcsec}^{2}\right)$ was cleaned. If a peak was found in this secondary image above a second SNR cut-off (the 'field SNR cut-off', for which SNR = 10 was adopted), a CLEAN box with a size 75 per cent of the fitted CLEAN beamsize was placed around the image and 25 iterations of the CLEAN algorithm were carried out with a gain of 0.05 . The CLEAN 
component signal was subtracted from the data, and the data inverted back into the $u-v$ plane, with the CLEAN procedure repeated until a residual cut-off level ( 5 per cent of the peak or $6 \sigma$ ) was reached. This means that we have a slightly higher sensitivity to secondary features within this smaller field around the primary component than in the larger search field, owing to the extreme taper applied to the visibility data input to the low-resolution search image (even accounting for the slightly lower SNR cut-off in the small field), and thus are somewhat more likely to find a faint source as a companion to a brighter source than on its own.

For each field identified and CLEANed, a phase-only selfcalibration with 10-s solution interval was performed and the CLEAN procedure was repeated. The CLEAN model was saved, and a modelfitting procedure (DIFMAP MODELFIT) was run for five iterations on the $u-v$ data. The starting point for model-fitting was the CLEAN boxes found and stored during the iterative CLEANing described above, with a Gaussian component assigned to each distinct CLEAN box (boxes must be further than 0.2 arcsec apart). The data products of the automapping procedure outlined above are the CLEAN model, the set of CLEAN windows, the list of MODELFIT components, and the log-file generated by DIFMAP containing the commands executed plus output information from the noise tests (this log-file can be executed as a script in DIFMAP reproducing the original results).

We have adopted the Gaussian MODELFIT results as the best representation of the DIFMAP analysis. Each component has a best-fitting position, major axis size, elliptical Gaussian axis ratio and position angle. In addition, uncertainties for the fitted quantities are evaluated from the covariance matrix. These quantities can then be filtered to select candidates with multiple components, and further filtered to find those with multiple compact components. Note that once distinct CLEAN boxes are laid down by the automapper, a component is necessarily created that will be passed to the model-fitting. The model-fitting procedure may then move this component where it pleases, possibly inside the exclusion box of 0.2 arcsec, or possibly outward to arbitrarily large separations. There is no provision for deleting poorly constrained components during model-fitting, and these must be dealt with during the analysis phase. For example, a moderate flux density cut-off of $2 \mathrm{mJy}$ per component, which corresponds to $5 \sigma$ for good data taken with $50-\mathrm{MHz}$ bandwidth, is found to reject most of the extraneous components.

The limitations of this algorithm include a restricted search field (only 256 arcsec square around the pointing centre in the inner quarter of the large image), a relatively conservative search SNR limit ( $8 \sigma$ to avoid many spurious detections) in this large field, and dynamic range problems (often spurious multiple components are found on sidelobes from bright sources). However, the automatic mapping has proved to be a reliable method of finding the best candidates quickly.

In some cases the automatic mapping program finds spurious structure or sources. Without manual intervention it is difficult to find the cause of many of these anomalies. However, the common symptom of such problems is that the final peak flux density after CLEANing and self-calibration is much greater than the initial peak flux density. The ratio of these two numbers, the 'reliability number' $(\mathrm{RN})$, should theoretically be around 1 . In practice we have found that maps with $\mathrm{RN}>1.5$ usually indicate a data problem, and $\mathrm{RN}=2$ almost certainly indicates a spurious detection.

Because the goal of CLASS is to identify lens systems with multiple compact components, it is crucial that we evaluate the effectiveness of our automapper in finding such objects. To this end, we constructed fake visibility data sets by adding pairs of point components (using the AIPS task UVSUB) to actual CLASS fields in which

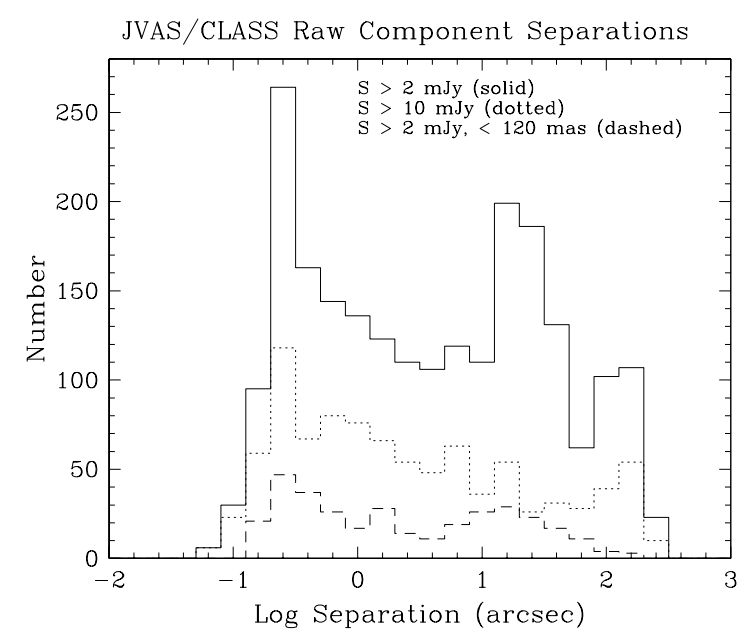

Figure 1. Histogram of maximum component separations for the 2219 CLASS sources that have at least two components with individual flux densities of $2 \mathrm{mJy}$ or more (solid). Also shown are cuts at $10 \mathrm{mJy}$ on component flux densities for 938 sources (dotted), and with component sizes of 120 mas or smaller for 333 sources (dashed).

no sources were detected by the VLA. This was repeated over a grid of component separations (0.15-10 arcsec), total flux densities (30$100 \mathrm{mJy})$ and flux density ratios (1:1-20:1) to simulate two-image gravitational lens systems. The pairs were randomly oriented on the sky and placed up to 20 arcsec from the field centre to mimic pointing inaccuracies. The data were then passed through the automapper, and the resulting MODELFIT output was analysed. We find that for separations $\gtrsim 300$ mas, the automapper consistently picks up two compact components when the fainter component has flux density $>2 \mathrm{mJy}$. For a source with $30 \mathrm{mJy}$ of total flux density, this corresponds to a flux density ratio of approximately $15: 1$. As expected, the identification of multi-component sources with larger total flux density is complete to larger flux density ratios. The automapper becomes increasingly inefficient at identifying multiple components as their separation decreases below 300 mas. At 150-mas separation, almost all of the data sets are fitted to a single component, the major axis of which increases with decreasing flux density ratio.

Finally, there were found to be minor irregularities in the automapping for multiple-component sources, with extraneous components being generated because of the high sidelobes of the VLA snapshot beam, particularly sidelobes from a source outside the small (70 arcsec) field. This effect can be seen in the histogram of maximum component separations in the full sample which is shown in Fig. 1. The secondary peak at around 20 arcsec is almost certainly due to sidelobes, as the dirty beam for a VLA snapshot has its main sidelobes, which have an amplitude of 40 per cent of the peak, in a hexagonal pattern spaced at 20 arcsec around the centre. This was not deemed to be a serious problem for the lens survey, as no true multiple sources would be lost, and simple filtering on the flux density and/or size of the MODELFIT components will remove most of these cases, as shown by the dotted and dashed curves in Fig. 1. However, the reader must be warned that the automatically generated catalogue still contains these spurious sources.

\section{CLASS RESULTS AND STATISTICS}

In Table 3 we show the first page of the JVAS/CLASS results data base. The entire sample is available from $\mathrm{CDS},{ }^{1}$ and as an ASCII

${ }^{1}$ http://www.cdsweb.u-strasbg.fr 
Table 3. First page of CLASS catalogue. Column 1 (bytes 0-16): GB6J name from the GB6 catalogue (Gregory et al. 1996); column 2 (18-25): name in observing list; column 3 (27-30): reliability number of map (see text); NOSH indicates a non-detection; column 4 (32-42): pointing position RA (J2000); column 5 (46-55): pointing position Dec. (J2000); column 6 (60-70): observing epoch (90-92= JVAS, $94=$ CLASS1, $95=$ CLASS2, $98=$ CLASS3, 99 = CLASS4); column 7 (74-86): detected component position(s) RA (J2000); column 8 (89-101): detected component position(s) Dec. (J2000); column 9 (103-111): 8.46-GHz flux density (mJy) of component(s); column 10 (113-118): fitted major axis (mas) of component(s); column 11 (120-123): fitted axial ratio of component(s); column 12 (125-128): fitted position angle (degrees) of component(s).

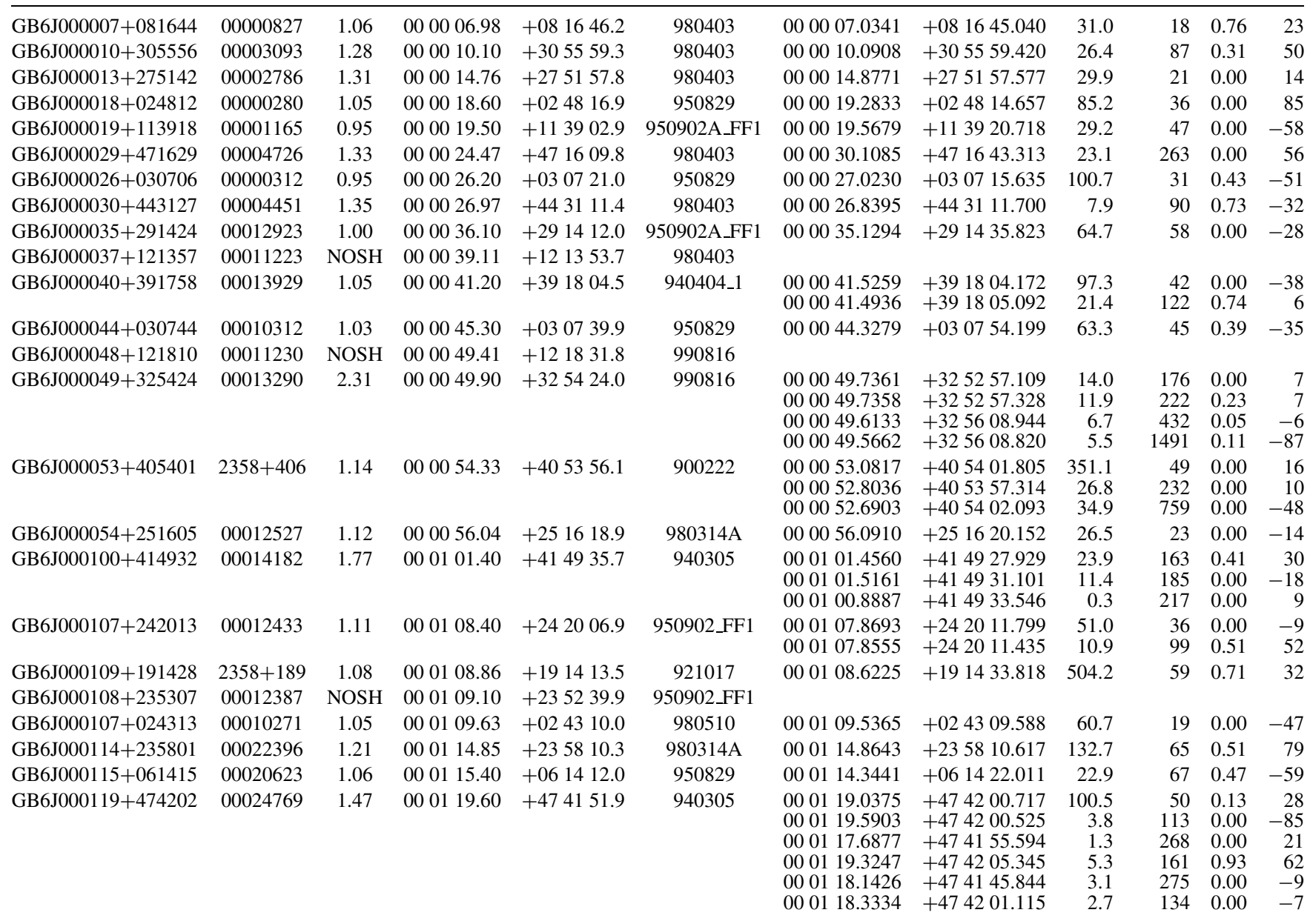

text file from http://www.jb.man.ac.uk/research/gravlens. This table contains the raw output from the automapping, and does not include manual re-mapping of questionable cases. Note that the last six columns contain the model-fitting information for position, flux density, size and orientation of the Gaussian components. The axial ratio is often found to be zero, which indicates a component that it thinks is unresolved in one dimension. If the major axis is small (150 mas or less), then this indicates an unresolved component, while if the major axis is significantly larger than this, then a zero or very small axial ratio usually indicates a poorly fitted component. These pathological cases are most often seen in cases where a large number of spurious and usually weak components are fitted to unCLEANed sidelobes on poorly calibrated data. For these objects, manual imaging, self-calibration and more careful model-fitting are being carried out. The reader should be careful in interpreting the automapping output for sources with more than two components, for this reason.

Fig. 2 shows a histogram of the $4.85-\mathrm{GHz}$ flux densities of the complete sample taken from the GB6 survey. The 30-mJy lower flux density limit is clearly visible. Also shown in Fig. 2 is the distribution of measured $8.46-\mathrm{GHz}$ flux densities for detections in the complete sample. Sources generally have less detected flux density at $8.46 \mathrm{GHz}$ than the $4.85-\mathrm{GHz}$ survey flux density. This is for a combination of reasons, but mainly spectral index and the fact that some of the sources are extended and partially resolved with the VLA in A-configuration.

Histograms of the GB6 versus NVSS spectral indices and CLASS versus GB6 are plotted in Fig. 3 for those sources in the complete sample that were detected in CLASS. The spectral index distribution derived using the observed 8.46-GHz-band merged flux densities and the $4.85-\mathrm{GHz}$ GB6 catalogue values is rather broad, centred around $\alpha \approx-0.6$, with a long tail steepward. This behaviour might reflect turnover in the spectra, source variability, resolution effects, a Malmquist-like bias, or probably a combination of most of the above. In particular, near the cut-off GB6 flux density the distribution will probably be dominated by sources scattered upward in flux density into the sample owing to variability or measurement error over and above those lost downward out of the sample - this is the Malmquist bias effect. In addition, steeper spectrum sources tend to be extended and thus are more likely to be resolved in the highresolution CLASS observations and to have depressed $8.46-\mathrm{GHz}$ flux densities, further steepening their apparent integrated spectrum. 


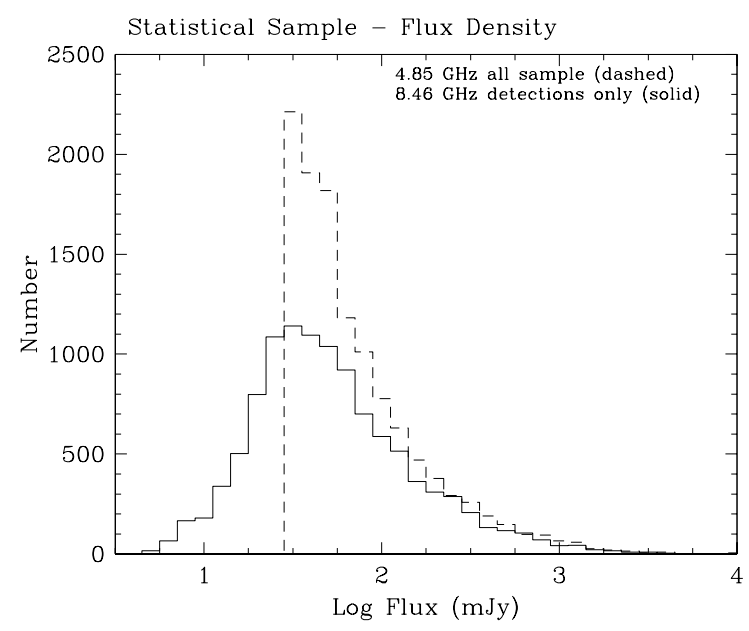

Figure 2. Histogram of CLASS 8.46-GHz flux densities for detections in the complete sample with components merged (solid) and GB6 4.85-GHz flux densities for the complete sample (dashed). The GB6 flux density limit of $30 \mathrm{mJy}$ is easily seen in the dashed histogram.

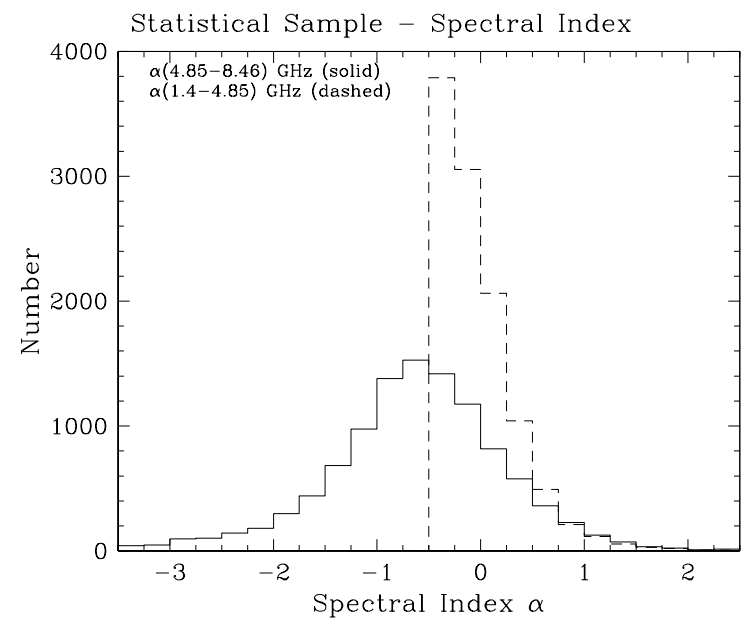

Figure 3. Histogram of spectral indices between CLASS and GB6 (solid) and between GB6 and NVSS (dashed). Sources from the complete sample detected in CLASS at $8.46 \mathrm{GHz}$ are plotted (with any multiple components merged). The $\alpha \geqslant-0.5$ selection between 1.4 and $4.85 \mathrm{GHz}$ is clearly seen in the dashed histogram.

In Fig. 4 the measured 8.46-GHz flux densities are plotted versus the GB6 flux density for the complete sample. The long tail toward steep spectral indices $\alpha<-0.5$ and non-detections in CLASS are clearly shown. A similar plot of CLASS/GB6 (8.46 to $4.85 \mathrm{GHz})$ versus GB6/NVSS (4.85 to $1.4 \mathrm{GHz}$ ) spectral indices is shown in Fig. 5. The spread in CLASS/GB6 spectral index is large. Furthermore, those sources with highly inverted $(\alpha>1.5)$ spectra between GB6 and NVSS tend to have moderate CLASS/GB6 indices. The CLASS non-detections are again shown as a bar at the bottom, and these are predominantly sources with GB6/NVSS spectral index near the cut-off, although there is a significant tail of sources with inverted low-frequency spectra. The CLASS/GB6 spectral index is plotted versus GB6 flux density in Fig. 6. The trend toward larger scatter in spectral index with lower GB6 flux density is clearly seen. The upper envelope with inverted spectra concentrated to lower flux densities at the lower frequency band is expected; however, the excess of steep-spectrum sources at the lower flux densities is worrisome, and probably related to the problem of non-detections. Finally, the 8.46- to $1.4-\mathrm{GHz}$ NVSS spectral index is plotted versus the NVSS flux density in Fig. 7. The spectral index range is tightened up considerably, and the envelopes arising from selection are clearly seen with the non-detections clustering at the limit for the spectral and flux density cut-offs. The 'cleaner' nature of this relation is probably due to being well above the NVSS survey flux density limit (reducing bias), the fact that the CLASS and NVSS surveys are nearly contemporaneous, and the intrinsic reduction in variability effects at the lower NVSS frequency.

There is a known increase in the rms uncertainty in GB6 flux densities at lower declination, with the $5 \sigma$ limit $S_{0}$ increasing from less than $20 \mathrm{mJy}$ at declination $\delta=+20^{\circ}$ to over $30 \mathrm{mJy}$ at the equator (Gregory et al. 1996). To investigate this effect, we plot the histograms of the CLASS/GB6 spectral index, splitting the sample at declination $+20^{\circ}$ (Fig. 8). There is a slight widening of the distribution and shift of the centroid toward steeper spectral index in the lower declination sub-sample, consistent with the effect. This points toward problems with GB6 flux densities near the selection cut-off, especially as we see a significantly increased non-detection rate at lower declinations also (see below).

\subsection{Detection rate}

The statistics of detections and multiple components found in the JVAS/CLASS sessions are given in Table 2. Of the 16503 targets observed, 14593 were detected in the automapping stage (88.4 per cent).

In Fig. 9 we display the percentage of observed sources that were not detected in the complete sample, as a function of 5-GHz flux density and spectral index. It can be clearly seen that the detection rate drops with both decreasing flux density and steeper spectra, as expected. In the survey as a whole, the non-detection rate is much higher (11.6 per cent) than in the complete sample (6.7 per cent). The extra non-detections arise from a variety of reasons which have already been described. The main extra contribution is likely to arise from parts of the survey where the spectral selection was relaxed or absent (particularly parts of CLASS-1). This is likely to have caused significantly more contamination by steep-spectrum and resolved sources. In addition, a few of the inverted-spectrum non-detections found at lower Galactic latitudes are identified as planetary nebulae.

The 6.7 per cent non-detection rate in the complete sample is relatively low: none the less, a $30-\mathrm{mJy}$ source at $5 \mathrm{GHz}$ should be detectable at $>20 \sigma$ at $8.46 \mathrm{GHz}$, even with a moderately steep radio spectrum. If we split the complete sample at declination $+20^{\circ}$ as we did earlier (see Fig. 8), we find that for $\delta>20^{\circ}$ the non-detection rate is only 3.8 per cent, and rises to 10.4 per cent for $\delta<20^{\circ}$. Again, this points toward problems related to the GB6 flux density scale near the cut-off, as the GB6 rms flux density uncertainty rises sharply at low declinations. Note that the non-detection rate for GB6 flux densities above $60 \mathrm{mJy}$ drops to 2 per cent overall (46 per cent of the total sample is above this cut-off).

Of the non-detections, 100 are within the existing regions of the FIRST survey (Becker, White \& Helfand 1995) and can be investigated in detail. Just over half of the non-detections (53/100) are due to classical double radio sources, in which one of the radio lobes is not picked up within the 70-arcsec search radius in the GB6/NVSS correlation (if the second lobe were included then the source would be dropped from the sample as the combined spectral index would pass the cut-off). Of the remaining 47 sources, 21 are revealed as 


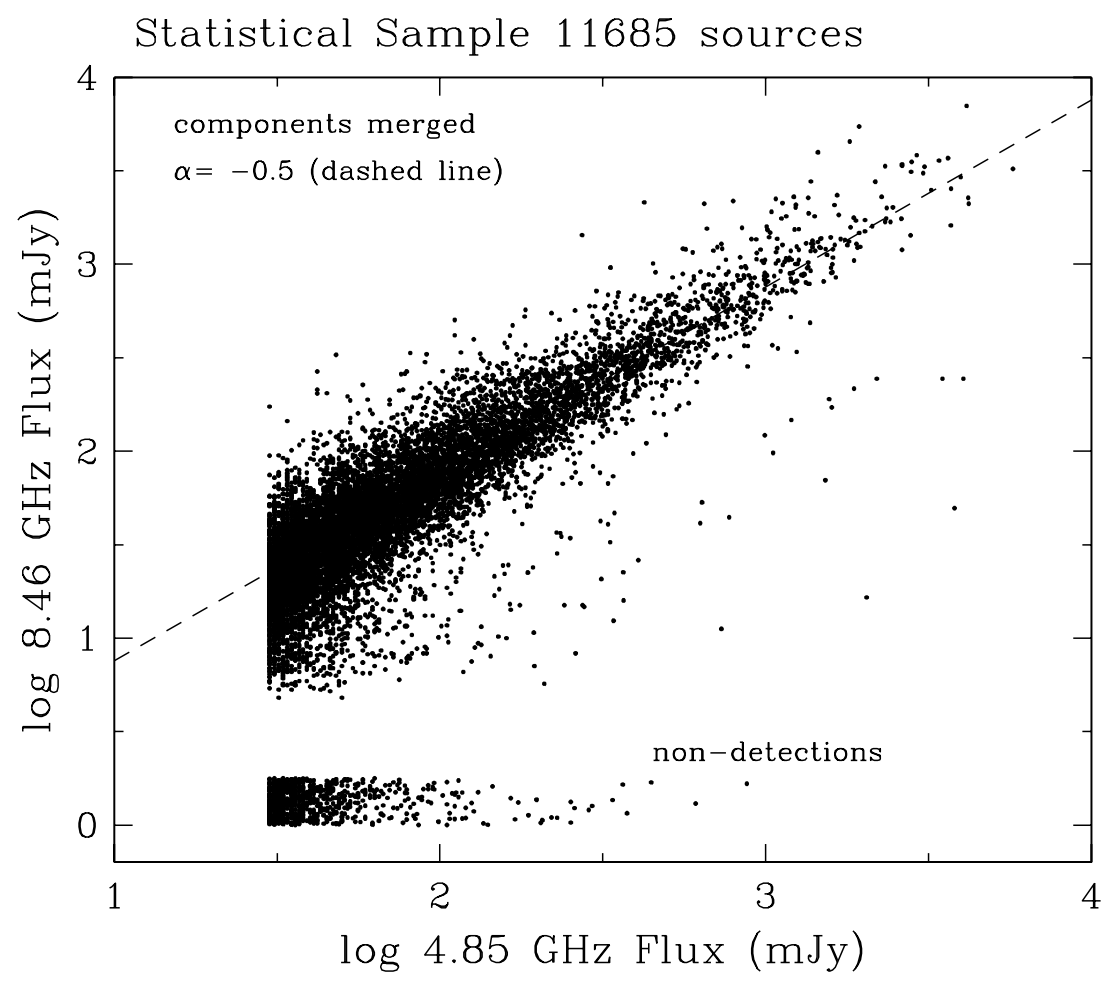

Figure 4. Observed JVAS/CLASS 8.46-GHz flux density versus GB6 4.85-GHz flux density, for the complete sample. For sources found with multiple components, the merged $8.46-\mathrm{GHz}$ flux densities were used. Sources above the dashed line have apparent spectral indices between CLASS and GB6 flatter than $\alpha \geqslant-0.5$. The bar of sources at the bottom represents GB6 targets where no CLASS source was detected (the assigned 8.46-GHz flux density is arbitrary and randomly spread to show the distribution with $4.85-\mathrm{GHz}$ flux density).

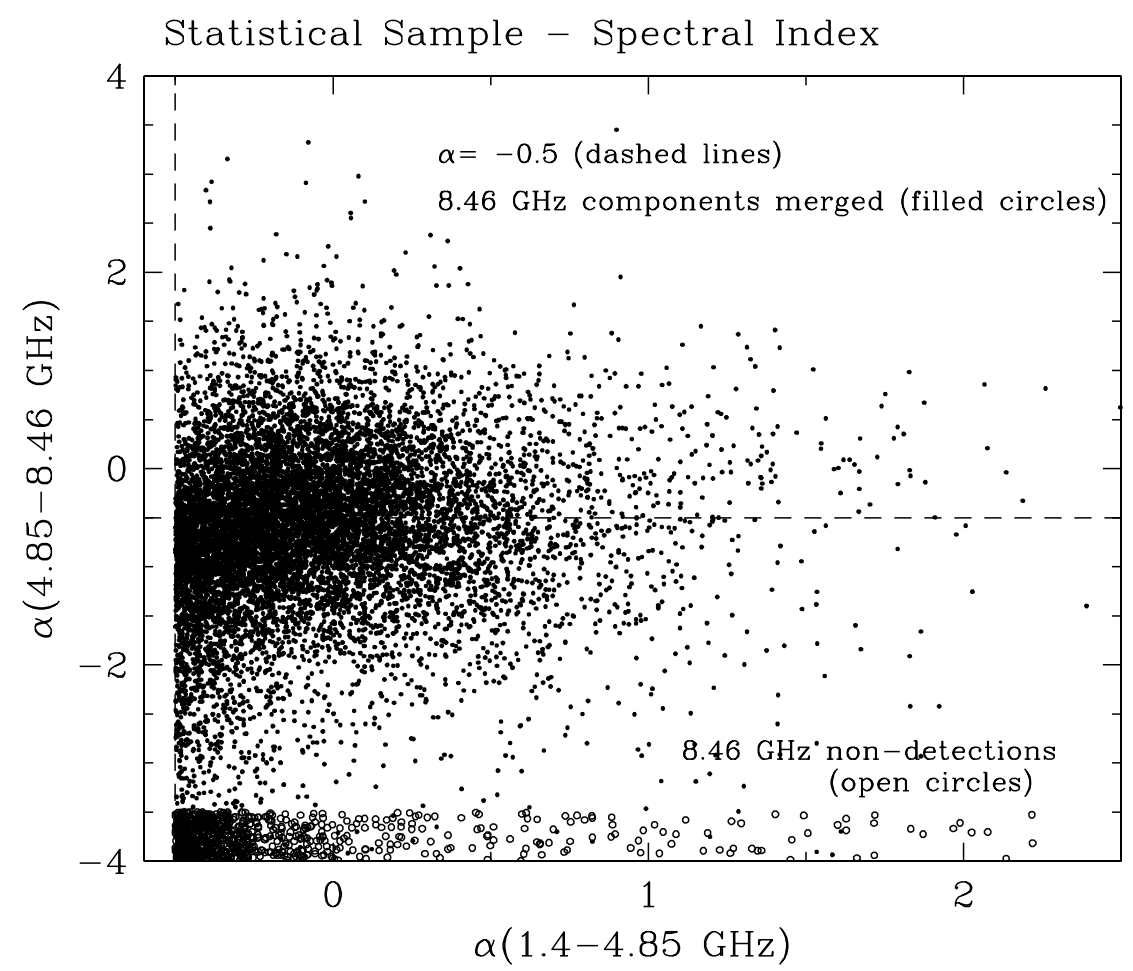

Figure 5. Spectral indices between CLASS and GB6 ( 8.46 and $4.85 \mathrm{GHz})$ plotted versus the GB6 to NVSS ( 4.85 and $1.4 \mathrm{GHz})$ index for the complete sample. As before, multiple components are merged. The bar of open circles at the bottom represents sources in the sample for which no 8.46-GHz detection was made, plotted versus the selection spectral index ( $y$-values randomly spread to show the distribution). 


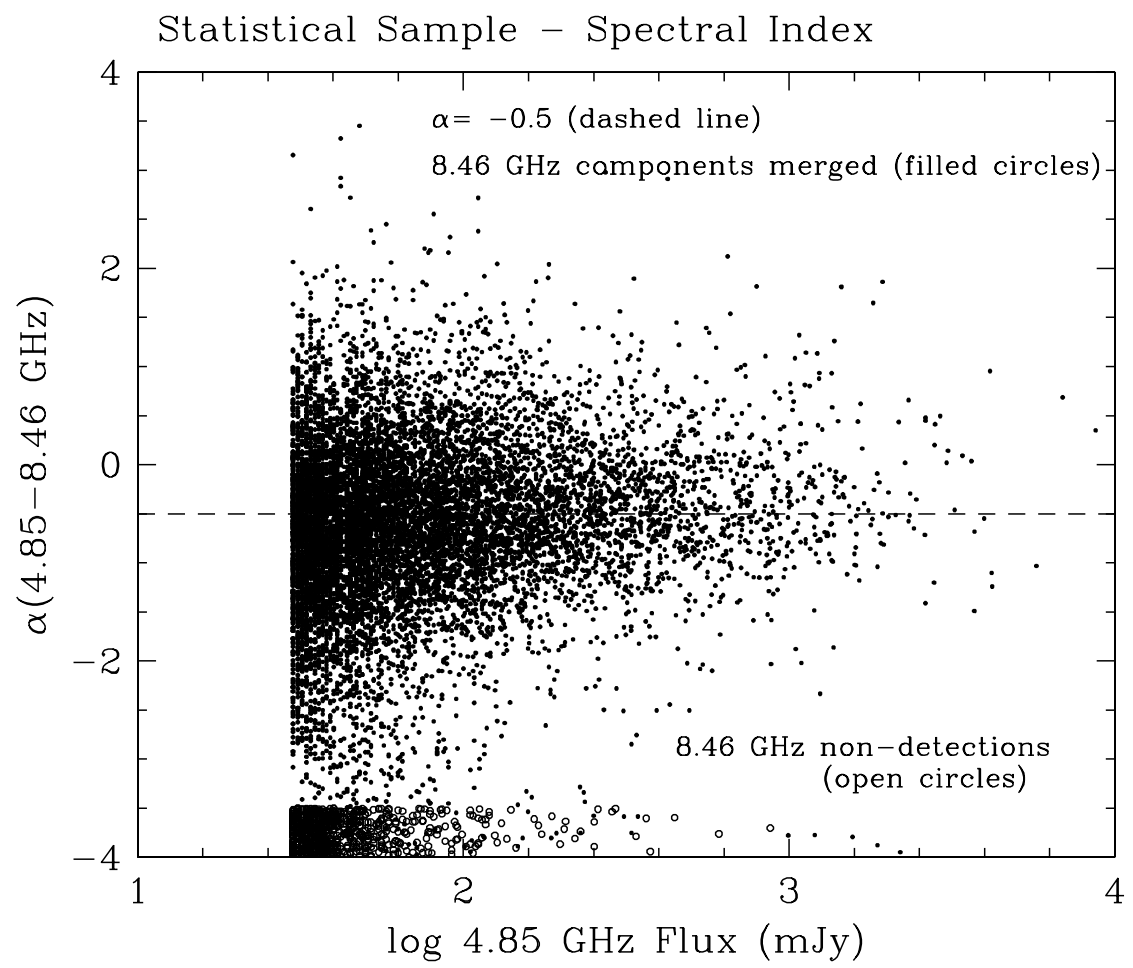

Figure 6. Spectral indices between CLASS and GB6 (8.46 and $4.85 \mathrm{GHz})$ plotted versus GB6 4.85-GHz flux density for the complete sample. As before, the bar of of open circles at the bottom represents sources in the sample for which no $8.46-\mathrm{GHz}$ detection was made.

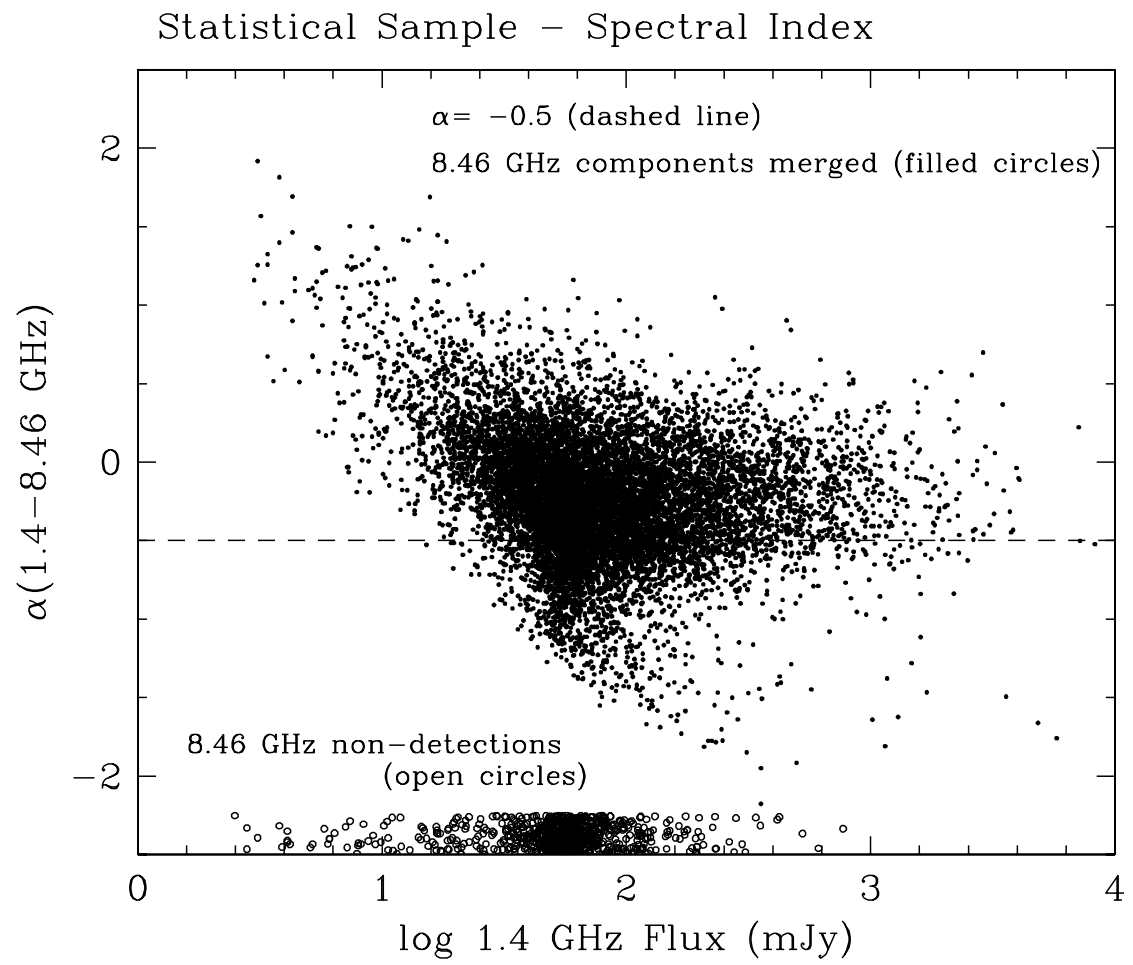

Figure 7. Spectral indices between CLASS and NVSS ( 8.46 and $1.4 \mathrm{GHz}$ ) plotted versus NVSS 1.4-GHz flux density for the complete sample. As before, the bar of of open circles at the bottom represents sources in the sample for which no 8.46-GHz detection was made.

classical double sources by FIRST. These are either single NVSS identifications, in the case of doubles with separation $<45$ arcsec, or more rarely larger sources; in either case they appear in the CLASS survey because the overall spectral index falls just under the $\alpha_{1.4}^{4.85}$ cut. 26 sources remain. Of these, eight are obviously large lobes or extended structure in which individual hotspots have registered in NVSS, resulting in inclusion in the CLASS sample. For 18 sources there is no obvious explanation for why they were not detected at 


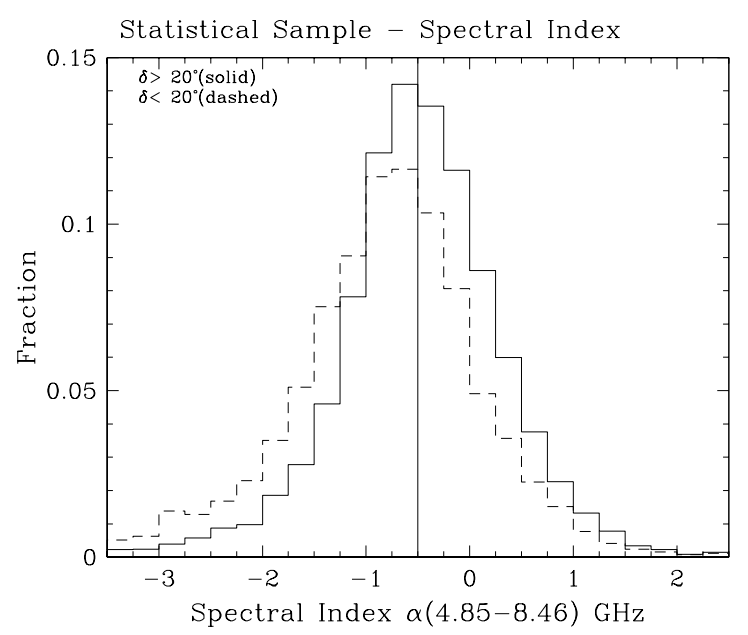

Figure 8. Histogram of spectral indices observed between CLASS and GB6 as a function of declination: Dec. $>20^{\circ}$ (solid) and Dec. $<20^{\circ}$ (dashed). The vertical axis is the fraction of the total falling in that bin. Only sources from the complete sample with CLASS detections are plotted (with any multiple components merged).
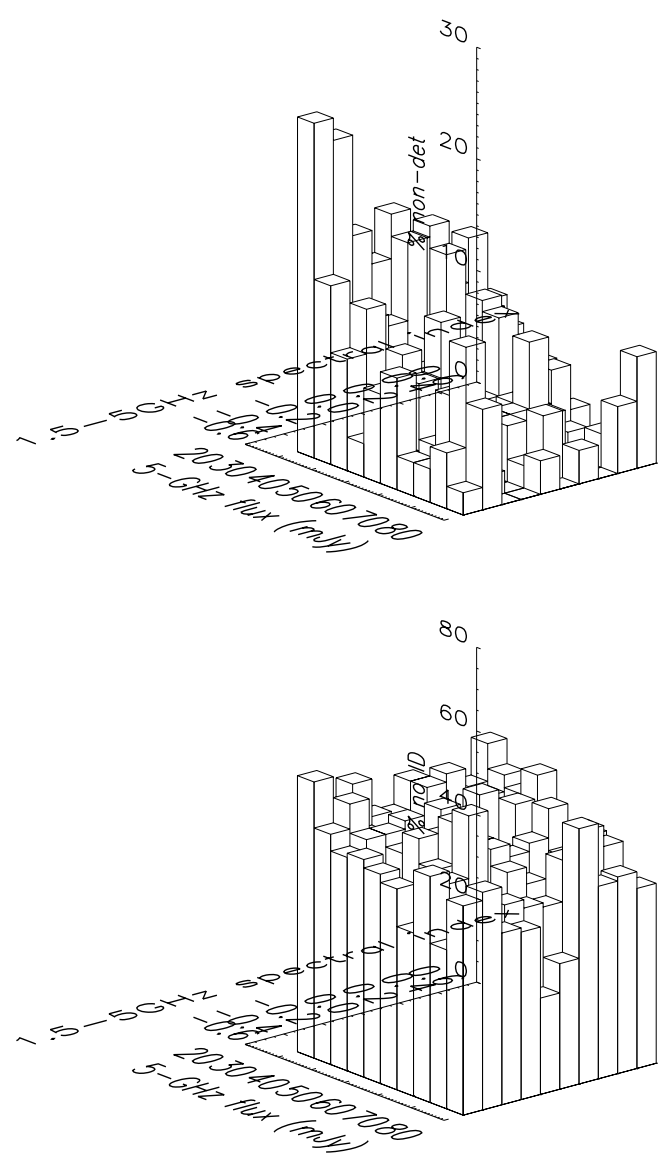

Figure 9. Rates of (top) non-detections in 8.46-GHz observations in the full JVAS/CLASS sample, and (bottom) sources without optical identifications, in the smaller statistically complete sample, plotted as a function of 1.4$4.85 \mathrm{GHz}$ spectral index and $4.85-\mathrm{GHz}$ flux density. Note the strong dependence of $8.46-\mathrm{GHz}$ detection probability on both radio spectral index and flux density, whereas the optical detection rate appears to depend mainly on radio flux density and decreases more gently.
$8.46 \mathrm{GHz}$; they generally have spectra approaching the spectral index cut, but are unresolved or only slightly resolved by the FIRST survey, and six of these 18 sources have a single NVSS identification and are unresolved by FIRST. However, these unexplained nondetections are most likely to be very small, relatively flat-spectrum double sources, or highly variable flat-spectrum sources, or sources for which the observations failed (although there is no evidence for the latter). In general, therefore, we can state that the contamination of the survey by intrinsically steep-spectrum sources is about 4-5 per cent, and about 1.5 per cent of the survey sources are not detected for reasons that are not clear.

\subsection{Statistics of the northern CLASS-1 region}

As mentioned in Section 2.1, the Texas 325-MHz survey only covered the region $\delta<71.6$ and thus north of this declination there was effectively no spectral selection in CLASS-1a. There were 489 87GB-selected sources that were observed in this manner, out of a total of 533 sources in $87 \mathrm{~GB}$ in this region with a flux density of $50 \mathrm{mJy}$ or higher, with the remaining 44 sources having been previously detected in other surveys. These 533 sources form a complete sample free of spectral selection that can be used to check the efficiency of our selection criteria in filtering out unresolved or complex sources.

Of the 489 CLASS targets in the northern region, 350 were detected in the automatic mapping. Added to the 44 previous detections in the region, the total detection rate is 394/533 (73.9 per cent). This detection rate is significantly lower than the average of the entire CLASS survey ( 89 per cent). In addition, 155 of the 350 CLASS-detected sources were found to have multiple components (44.3 per cent), a significantly higher rate than the overall CLASS average (14.3 per cent of detected sources in the complete sample). These statistics are consistent with the expectations for the spectral selection, which is designed to reduce the number of extended and intrinsically complex sources in the sample.

\section{DISCUSSION AND SUMMARY}

In total, 15.4 per cent of the entire survey and 13.3 per cent of the complete sample (17.4 and 14.3 per cent of detected sources respectively) have multiple components identified in the automapping process. Some of these multiple sources, however, will be due to sidelobes and other artefacts of the automapping process. For further analysis we require an estimate of which sources are genuine multiple objects. For lens candidate selection, in particular, further constraints are applied in total $8.46-\mathrm{GHz}$ flux density, in primary-to-secondary flux density ratio and in component separation. In Fig. 1 we show the histogram of maximum component separations in the full sample (the complete sample shows essentially the same distribution). The survey is clearly sensitive to multiple components with separations from 160 mas to 300 arcsec, and thus our primary goal of identification of gravitational lens candidates is achievable. Note that in Fig. 1 double-lobe sources, knots within lobes, core-jet sources, superposition of unrelated sources, artefacts of the automapping process and real multiple-image lens systems are all mixed together, and lenses are a tiny fraction of the total number of multiples! When restricted to components with flux densities of $2 \mathrm{mJy}$ and above, 15.1 per cent of all sources detected and 11.6 per cent of detected sources in the complete sample were multiple - these numbers better represent the fraction of true multiples in the survey. Details of candidate selection are discussed in Paper II of this series. 
Optical identifications have been sought from scans of the Palomar Sky Survey prints carried out by Kibblewhite et al. (1984). As expected from earlier work, the identification fraction of flatspectrum sources to $R \sim 20$ decreases with decreasing flux density, reaching 50 per cent at $\sim 50 \mathrm{mJy}$ and decreasing further thereafter. Redshift surveys are in progress to identify sources at these flux density levels (Falco, Kochanek \& Muñoz 1998; Marlow et al. 2000). Fig. 9 shows the optical identification rate to this level; it can be seen that the decrease with 5-GHz flux density is monotonic, and does not depend strongly on radio spectral index.

In summary, we have presented radio observations of a complete sample of flat-spectrum radio sources with the VLA. A total of 16503 distinct target sources have been observed, of which 11685 form a complete sample with the properties $S_{5 \mathrm{GHz}}>30 \mathrm{mJy},|b| \geqslant$ $10^{\circ}, \alpha_{1.4}^{4.85} \geqslant-0.5$. Contamination by steep-spectrum sources is around 4 per cent in the complete part of the survey. Further papers in the series will discuss the selection of candidate gravitational lenses and astrophysical inferences from the statistics of this sample.

\section{ACKNOWLEDGMENTS}

STM was supported by a R. A. Millikan Fellowship while at Caltech from 1992 to 1995, and by an Alfred P. Sloan Fellowship at the University of Pennsylvania. The CLASS survey at Caltech was supported by NSF grant AST-9117100, and RDB acknowledges the additional support of NSF AST-9529170, AST-9900866 and NASA NAG 5-7007. This research was supported in part by the European Commission TMR Programme, Research Network Contract ERBFMRXCT96-0034 'CERES'. The National Radio Astronomy Observatory is operated by Associated Universities, Inc., under cooperative agreement with the National Science Foundation. Finally, but not least, we thank the staff of the VLA for their assistance during our observing runs, and for providing the instruments that made this work possible.

\section{REFERENCES}

Argo M. K. et al., 2003, MNRAS, 338, 957

Augusto P., Wilkinson P. N., 2001, MNRAS, 320, L40

Augusto P., Wilkinson P. N., Browne I. W. A., 1998, MNRAS, 299, 1159

Augusto P. et al., 2001, MNRAS, 326, 1007

Baars J. W. M., Genzel R., Pauliny Toth I. I. K., Witzel A., 1977, A\&A, 61, 99

Becker R. H., White R. L., Helfand D. J., 1995, ApJ, 450, 559

Biggs A. D., Browne I. W. A., Helbig P., Koopmans L. V. E., Wilkinson P. N., Perley R. A., 1999, MNRAS, 304, 349

Biggs A. D. et al., 2003, MNRAS, 338, 1084

Browne I. W. A., Wilkinson P. N., Patnaik A. R., Wrobel J. M., 1998, MNRAS, 293, 257

Browne I. W. A. et al., 2003, MNRAS, 341, 13 (Paper II, this issue)

Burke B. F., 1989, in Mellier Y., Fort B., Soucail G., eds, Gravitational Lensing. Springer-Verlag, Berlin, p. 127

Chae K.-H., Mao S., Augusto P., 2001, MNRAS, 326, 1015

Chae K.-H. et al., 2002, Phys. Rev. Lett., 89, 1301

Cohn J. D., Kochanek C. S., McLeod B. A., Keeton C. R., 2001, ApJ, 554, 1216

Condon J. J., Cotton W. D., Greisen E. W., Yin Q. F., Perley R. A., Taylor G. B., Broderick J. J., 1998, AJ, 115, 1693

Douglas J. N., Bash F. N., Bozyan F. A., Torrence G. W., Wolfe C., 1996, AJ, 111, 1945

Falco E. E., Kochanek C. S., Muñoz J. M., 1998, ApJ, 494, 7

Fassnacht C. D. et al., 1999a, AJ, 117, 658

Fassnacht C. D., Pearson T. J., Readhead A. C. S., Browne I. W. A., Koopmans L. V. E., Myers S. T., Wilkinson P. N., 1999b, ApJ, 527, 498
Fassnacht C. D., Xanthopoulos E., Koopmans L. V. E., Rusin D., 2002, ApJ, 581,823

Gregory P. C., Condon J. J., 1991, ApJS, 75, 1011

Gregory P. C., Scott W. K., Douglas K., Condon J. J., 1996, ApJS, 103, 427

Hewitt J. N., Turner E. L., Lawrence C. R., Schneider D. P., Brody J. P., 1992, AJ, 104, 968

Jackson N. et al., 1995, MNRAS, 274, L25

Jackson N. et al., 1998, MNRAS, 296, 483

Keeton C. R., Madau P., 2001, ApJ, 549, L25

Kibblewhite E. J., Bridgeland M. T., Bunclark P. S., Irwin M. J., 1984, in Klingles D. A., ed., Astronomical Microdensitometry Conference. NASA-2317, p. 277

King L. J., Browne I. W. A., Muxlow T. W. B., Narasimha D., Patnaik A. R., Porcas R. W., Wilkinson P. N., 1997, MNRAS, 289, 450

Kochanek C. S., 1995, ApJ, 445, 559

Kochanek C. S., 1996, ApJ, 473, 595

Koopmans L. V. E., de Bruyn A. G., 2000, A\&A, 358, 793

Koopmans L. V. E., Fassnacht C. D., 1999, ApJ, 527, 513

Koopmans L. V. E. et al., 1998, MNRAS, 303, 727

Koopmans L. V. E., de Bruyn A. G., Xanthopoulos E., Fassnacht C. D., 2000a, A\&A, 356, 391

Koopmans L. V. E. et al., 2000b, A\&A, 361, 815

Marchã M. J., Caccianiga A., Browne I. W. A., Jackson N., 2001, MNRAS, 326, 1455

Marlow D. R. et al., 1999, AJ, 118, 654

Marlow D. R., Rusin D., Jackson N., Wilkinson P. N., Browne I. W. A., Koopmans L. V. E., 2000, AJ, 119, 2629

Marlow D. R. et al., 2001, AJ, 121, 619

Myers S. T. et al., 1995, ApJ, 447, L5

Myers S. T. et al., 1999, AJ, 117, 2565

Patnaik A. R., Browne I. W. A., Wilkinson P. N., Wrobel J. M., 1992a, MNRAS, 254, 655

Patnaik A. R., Browne I. W. A., Walsh D., Chaffee F. H., Foltz C. B., 1992b, MNRAS, 259, 1

Patnaik A. R., Porcas R., Browne I. W. A., 1995, MNRAS, 274, L5

Phillips P. M. et al., 2000, MNRAS, 319, L7

Phillips P. M. et al., 2001, MNRAS, 328, 1001

Rengelink R. B., Tang Y., de Bruyn A. G., Miley G. K., Bremer M. N., Roettgering H. J. A., Bremer M. A. R., 1997, A\&AS, 124, 259

Rusin D., Ma C.-P., 2001, ApJ, 549, L33

Rusin D., Tegmark M., 2001, ApJ, 553, 709

Rusin D. et al., 2001a, AJ, 122, 591

Rusin D. et al., 2001b, ApJ, 557, 594

Rusin D., Norbury M., Biggs A. D., Marlow D. R., Jackson N. J., Browne I. W. A., Wilkinson P. N., Myers S. T., 2002, MNRAS, 330, 205

Shepherd M. C., 1997, in Hunt G., Payne H. E., eds, ASP Conf. Ser. Vol. 125, Astronomical Data Analysis Software and Systems VI. Astron. Soc. Pac., San Francisco, p. 77

Shepherd M. C., Pearson T. J., Taylor G. B., 1994, BAAS, 26, 987

Snellen I. A. G., de Bruyn A. G., Schilizzi R. T., Miley G. K., Myers S. T., 1995, ApJ, 447, L9

Snellen I. A. G., Mack K.-H., Tschager W., Schilizzi R., 2000, in Conway J., Polatidis A., Booth R., eds, Proc. 5th EVN Symposium. Onsala Space Observatory, Chalmers Technical University, Gothenburg, Sweden

Snellen I. A. G., McMahon R. G., Dennett-Thorpe J., Jackson N., Mack K.-H., Xanthopoulos E., 2001, MNRAS, 325, 1167

Snellen I. A. G., McMahon R. G., Hook I. M., Browne I. W. A., 2002, 329, 700

Sykes C. M. et al., 1998, MNRAS, 301, 310

Turner E. L., Ostriker J. P., Gott J. R., 1984, ApJ, 284, 1

White R. L., Becker R. H., 1992, ApJS, 79, 331

Wilkinson P. N., Browne I. W. A., Patnaik A. R., Wrobel J. M., Sorathia B., 1998, MNRAS, 300, 790

Winn J. N. et al., 2000, AJ, 120, 2868

Xanthopoulos E. et al., 1998, MNRAS, 300, 649

This paper has been typeset from a $\mathrm{TE}_{\mathrm{E}} \mathrm{X} / \mathrm{L} \mathrm{T}_{\mathrm{E}} \mathrm{X}$ file prepared by the author. 